\title{
Inversion of refraction data by wave field continuation
}

\author{
Robert W. Clayton* and George A. McMechan广
}

\begin{abstract}
The process of wave equation continuation (migration) is adapted for refraction data in order to produce velocity-depth models directly from the recorded data. The procedure consists of two linear transformations: a slant stack of the data produces a wave field in the $p-\tau$ plane which is then downward continued using $\tau=0$ as the imaging condition. The result is that the data wave field is linearly transformed from the time-distance domain into the slowness-depth domain. where the velocity profile can be picked directly. No traveltime picking is involved, and all the data are present throughout the inversion.

The method is iterative because it is necessary to specify a velocity function for the continuation. The solution produced by a given iteration is used as the continuation velocity function for the next step. Convergence is determined when the output wave field images the same velocity-depth function as was input to the continuation.

The method obviates the problems associated with determining the envelope of solutions that are consistent with the observations, since the time resolution in the data is transformed into a depth resolution in the slowness-depth domain.

The method is illustrated with several synthetic examples, and with a refraction line recorded in the Imperial Valley, California.
\end{abstract}

\section{INTRODUCTION}

Refraction profiles are conventionally analyzed by extracting traveltime information from the data and performing a WiechertHerglotz integration to produce a velocity-depth profile. We present an alternate approach to refraction inversion which consists of transforming the entire data wave field into the slowness-depth domain. The process involves two linear transformations: a slant stack followed by a downward continuation.

Applications of slant stacking to reflection data were first demonstrated by Schultz (1976) and Schultz and Claerbout (1978). Examples of the processing of refraction wave fields by slant stacking were presented by McMechan and Ottolini (1980), Stoffa and Buhl (1979), and Phinney et al (1980). The result of this transformation is a wave field in the ray parameter-time intercept $(p-\tau)$ plane. The image that forms in the $p-\tau$ plane is the "tau" curve (cf., Bessonova et al, 1976), required for Wiechert-Herglotz inversion. Chapman (1978) showed that the inverse slant stack was a general method of solving the forward problem and therefore the slant stack was a useful start to the inverse problem.

Recently, Garmany et al (1979) showed that the inversion of a tau curve can be expressed in linear form if the integration is performed along lines of constant $p$. We employ a downward continuation method to transform linearly from the $p-\tau$ domain directly into the slowness-depth domain. Since both slant stacking and downward continuation transform the entire wave field. neither the traveltime curve nor the tau curve need be picked. Instead. picking is delayed until the last step when the slownessdepth model is extracted directly from the output wave field. The wave field transformation approach to inversion has the advantage that all the data contribute to the final image; there is no subjective selection of data (e.g., via traveltime picking) as in previous methods. In theory, since both downward continuation and slant stacking are reversible transformations, this approach could potentially be used to generate synthetic refraction gathers from the $p-z$ plane.

The downward continuation part of this technique is iterative because it is necessary to specify a velocity function. Convergence is achieved when the extracted velocity function is the same as that input to the continuation. In the examples we have tested, convergence was obtained in less than five iterations.

We present the theory for the inversion of refraction profiles by double transformation of the data wave field. The method is illustrated with several synthetic examples and with a line of refraction data recorded in the Imperial Valley, California.

For real data, the chief limitation on the method presented here is the ability to produce a reasonable slant stack of the data. The paucity and sometimes significant lateral variations that occur in typical refraction surveys will introduce artifacts into the slant stack. If the artifacts are too severe, then the applicability of the method is doubtful. Also, we make no attempt here to utilize the information contained in the subcritical reflections.

\section{THEORY}

The data recorded in a typical refraction experiment is in the format of a common shot gather. Making the assumption that velocity varies only with depth, it is equivalent to treat such data as a common midpoint (CMP) gather. We shall use symbols $t$ for

Manuscript received by the Editor February 21, 1980; revised manuscript received September $15,1980$.

* Stanford Exploration Project, Department of Geophysics. Stanford University. Stanford. CA 94305.

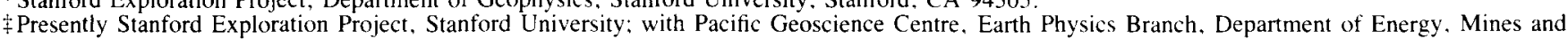
Resources, Box 6000, Sidney, B.C., V8L 4B2 Canada.

0016-8033/81/0601-860\$03.00. (C) 1981 Society of Exploration Geophysicists. All rights reserved. 

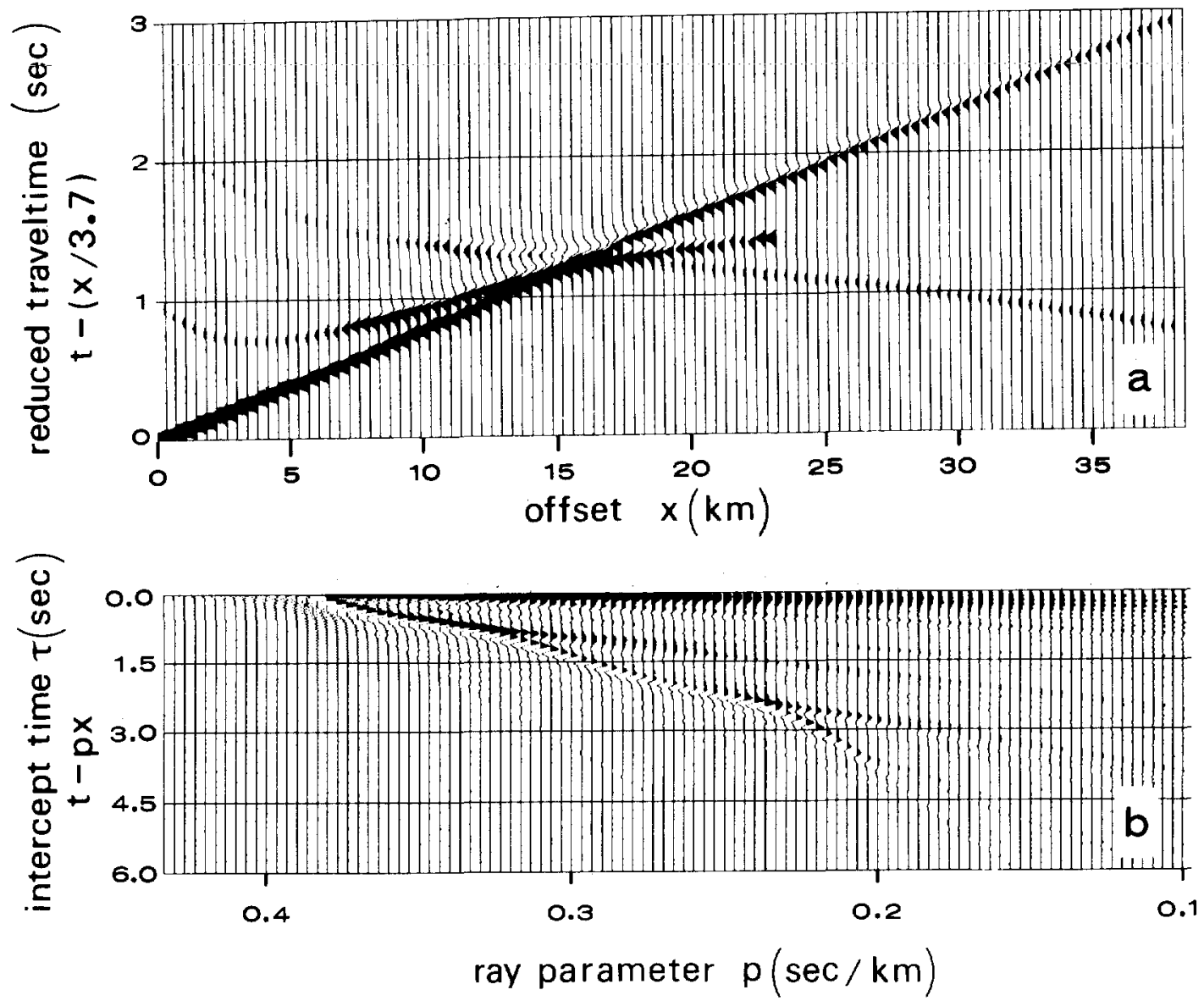

FIG. 1. The first transformation. The upper (a) half of this figure contains a synthetic refraction profile plotted in reduced time format. The reduction velocity is $3.7 \mathrm{~km} / \mathrm{sec}$. These data are transformed by slant stacking into the plane-wave decomposition shown in the lower (b) half of the figure. This transformation is the first half of the process of inversion of the data wave field. The result of downward continuing this slant stacked wave field (b) is shown in Figure 2.

traveltime and $h$ for half-offset in a CMP coordinate system. The inversion of an observed refraction wave field in these coordinates involves two linear transformations: a slant stack and a downward continuation. Each of these will now be considered in turn.

A slant stack is a linear operation that transforms a wave field in the $t-h$ domain into a wave field in the $p-\tau$ domain. Here, $p$ is the ray parameter (horizontal slowness), and $\tau$ is the vertical component of $t$ [ $t$ projected to zero offset along a line of slope $p$ through the point $(t, 2 h)]$. Slant stacking of refraction profiles has been discussed in detail by McMechan and Ottolini (1980), so only a brief summary is given here.

Slant stacking can be performed in either the time or frequency domain; however, in the time domain, variable trace spacing is easier to handle. A slant stacked wave field is produced from a common shot gather by (Gel'fand et al, 1966; Chapman, 1978; McMechan and Ottolini, 1980):

$$
S(\tau, p)=\int_{-\infty}^{+\infty} P(\tau+2 p h, h) d h,
$$

where $P$ is the observed (seismogram) wave field and $S$ is the transformed $(p-\tau)$ wave field. A correction for the frequency dependence of the stack is performed by convolving $S(\tau, p)$ with an operator of the form $H(t) t^{-1 / 2}$ (cf., Phinney et al, 1980). Equation (1) can be cast in the frequency domain by using the Fourier central slice theorem

$$
S(\omega ; p)=P(\omega,-2 \omega p) .
$$

In other words, the two-dimensional (2-D) Fourier transform of $P$ evaluated along the slice $-2 \omega p$ is the Fourier transform with respect to time of its projection $S(\tau, p)$. For a more detailed presentation of these concepts, see Bracewell (1956) and Bracewell and Riddle (1967).

The main result of slant stacking is that the observed wave field is decomposed into plane wave (fixed $p$ ) components, each of which can be downward continued separately. Figure la contains an example of a synthetic common shot gather, and Figure $1 \mathrm{~b}$ is the corresponding slant stack. The finite aperture of the data set and spatial aliasing both contribute to artifacts in the slant stack. Relatively dense sampling of the offset coordinate is required to produce a reasonable image in the $p-\tau$ domain (cf., McMechan and Ottolini, 1980). Also, coherency of source signatures is required if multiple shots are involved in the field survey.

The second transformation in this technique is a downward continuation of the slant stacked wave field. This step resembles a depth migration, except that in this case it is being applied in the offset domain rather than in the midpoint domain. When velocity varies only with depth $[v=v(z)]$, the downward continuation of the wave field observed at the surface $(z=0)$ to any 

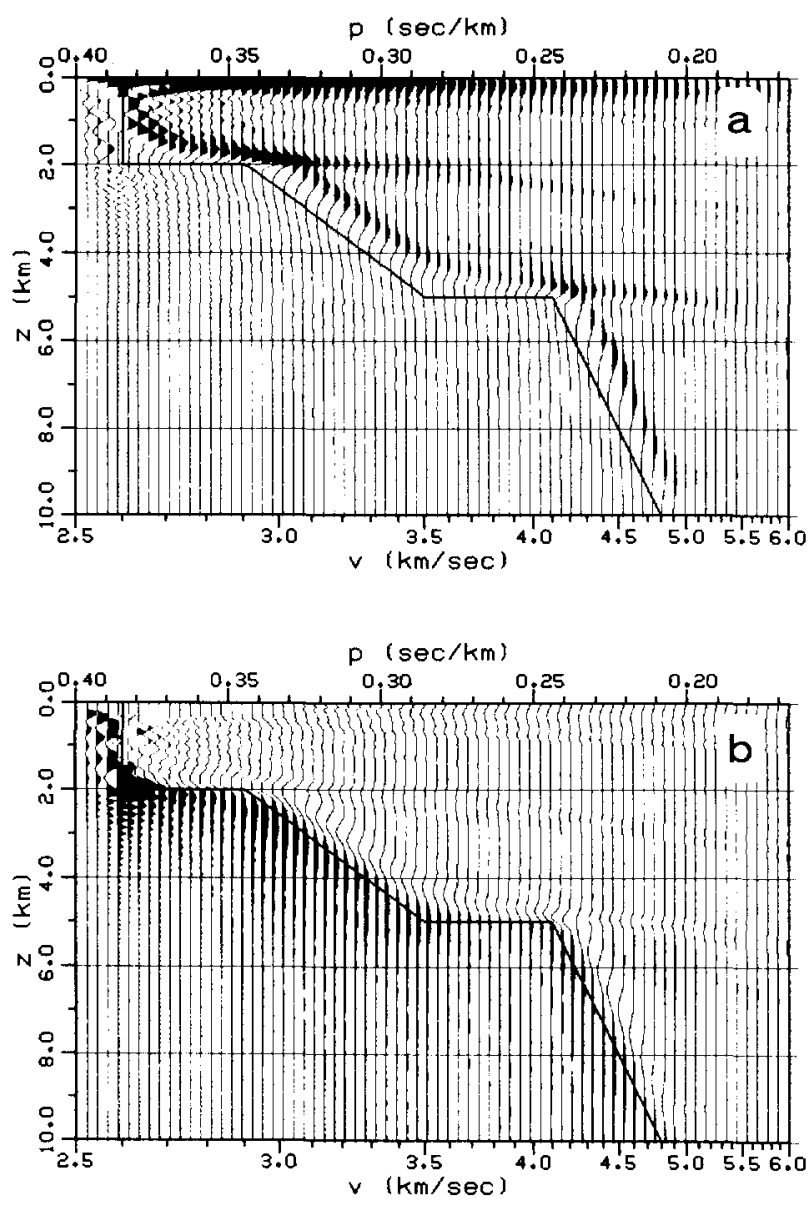

FIG. 2. The second transformation. The upper (a) half of this figure shows the result of downward continuing the slant stacked wave field in Figure 1b with the correct velocity-depth function (the solid line). The lower (b) half of this figure contains the same wave field as the upper, but with a phase rotation of $5 \pi / 4$. All the downward continued wave fields in the remainder of this paper are presented with this phase shift applied.

desired depth ( $z$ ) can be implemented by a phase rotation in the frequency domain (cf., Claerbout, 1976; Gazdag, 1978):

$P\left(\omega, k_{h}, z\right)=P\left(\omega, k_{h}, 0\right) \exp \left[-i 2 \int_{0}^{z}\left[\frac{\omega^{2}}{v^{2}(z)}-\frac{k_{h}^{2}}{4}\right)^{1 / 2} d z\right]$.

where $\omega$ is the temporal frequency and $k_{h}$ is the horizontal wavenumber (the dual of $h$ ). Equation (3) is an approximate solution to the wave equation

$$
\left[\frac{\partial^{2}}{\partial^{2} z}+\frac{\partial^{2}}{\partial^{2} h}+4 \frac{\omega^{2}}{v^{2}(z)}\right] P(\omega, h, z)=0 .
$$

The solution is approximate because amplitude terms depending on the logarithmic gradient of the velocity have been neglected. However, the traveltime aspects of the solution are correct. The first minus sign in equation (3) indicates that we are imaging upcoming waves.

To convert equation (3) into slowness form, substitute $-2 \omega p$ for $k_{h}$ :

$$
P(\omega,-2 \omega p, z)=P(\omega,-2 \omega p, 0) e^{-i \omega \Psi(p, z)},
$$

where

$$
\Psi(p, z)=2 \int_{0}^{z}\left[1^{2}(z)-p^{2}\right]^{1 / 2} d z
$$

Using relation (2), equation (5) may be rewritten in terms of slant stacked wave fields:

$$
S(\omega, p, z)=S(\omega, p .0) e^{-i \omega \Psi(p, z)} .
$$

The inverse Fourier transform of cquation (7) is

$$
S(\tau, p, z)=\int S\left(\omega, p,(1) e^{-i \omega|\Psi(p, z)-\tau|} d \omega .\right.
$$

With equation $(8)$, the surface $(z=0)$ slant stack can be extrapolated (downward continued) to ill depths. One could think of using this equation to fill out the entire $\tau-p-z$ space with data extrapolated from the surface. However, this is not necessary because the slowness image we seek lies on a plane in the $\tau-p-z$ space specified by the imaging condition. The desired image contains the bottoming points of all the plane-wave components. The plane which images this trajectory is specified by the condition $\tau=0$ because we wish to downward continuc each $p$ to the depth at which the corresponding ray bottoms. At that depth, $p$ of the ray equals the true slowness $\left(v^{-1}\right)$ of the medium for refractions and posteritical rellections. Precritical reflections image in a trajectory that splits of from the main slowness image at the critical reflection point. Setting $\tau=0$ in equation $(8)$ yields the desired result:

$$
s(p, z) \equiv S(0, p, z)=\int S(\omega, p, 0) e^{-i \omega \psi(p, z)} d \omega
$$

where $s(p, z)$ is defined as the slowness plane.

Implementation of equation (9) is straightforward. Each planewave component (each $p$ ) can be downward continued separately since $p$ enters equation (9) as a parameter. A computer program which realizes ( 9 ) consists basically of three nested loops: an outer one over $p$, then one over $z$, and an inner one over $\omega$. For some applications, it may be more efficient to cast equation (9) in the time domain ${ }^{1}$. Inverse transforming over $\omega$, we have

$$
s(p, z)=S[\tau-\psi(p, z), p, 0],
$$

which means that the slowness plane can be obtained by a normal moveout (NMO) correction applied to the slant stack.

One minor problem that occurs in the application of equation (9) is that $\Psi$ has a branch cut. Wo remedied this by altering the definition of $\Psi$ to

$$
\Psi(\omega, p, z)=2 \omega \int_{0}^{z}\left|\gamma^{2}(z)-p^{2}\right|^{1 / 2} d z .
$$

This stops the downward continuation from attenuating the wave field below depths where $p$ is greater than $v^{-1}$.

The application of equation (9) produces an image in the slowness plane. The image trajectory is composed of wavelets whose shapes are each defined by the phase shift associated with the reflection coefficient at each $z$. A refracted ray can be treated as having an effective reflection coefitient of $-i \operatorname{sgn}(\omega)$ ) (Chapman, 1978).

Figure $2 \mathrm{a}$ shows the $p-z$ wave field obtained by downward

\footnotetext{
${ }^{1}$ We thank the two reviewers of the paper for pointing out this modification In the examples presented, we implemented equation (9) in an array processor. The computation time is minor compared to the time it takes to plot the results. However, for implementation on a general purpose machine, equation (10) is probably faster.
} 
continuing the $p-\tau$ wave field in Figure $1 \mathrm{~b}$ using the $v(z)$ function (the solid line) superimposed on Figure 2a. This $v(z)$ function used for continuation is the same as that used to generate the synthetic seismograms (Figure 1a). The wave field in Figure 2 a should therefore image the corresponding $v(z)$ function exactly. The varying offset between the input $v(z)$ function and the image is due to the fact that there are phase shifts related to reflection coefficients which are not yet taken into account. Accurate location of the image trajectory is discussed below.

The object of inversion is to find the $v(z)$ [or, equivalently, in the present formulation, the $v^{-1}(z)$ ] function. However, the continuation step requires a velocity-depth function. Thus, obtaining a solution is necessarily an iterative process. Convergence is presumably to a unique model because there are no artificial constraints on the convergence path; the data set itself contains and provides the solution. The convergence criterion is that the output wave field images the same $v^{-1}(z)$ function that was input to the downward continuation, and this state can be detected by comparing the $p-z$ wave ficlds at successive iterations.

\section{SYNTHETIC EXAMPLES}

It is possible to implement wave field inversion in an interactive mode in which the slowness function extracted from the imaged wave field at each step is used as the continuation velocity for the next iteration. In this section we present a detailed example of inversion in an interactive mode. An example of automated convergence is included, but the production of a totally automatic inversion scheme that is able to adapt to a variety of conditions is far beyond the scone of this paper.

The most important aspect of inversion by wave field imaging is the determination of the location of the desired slowness trajectory in the downward continued $(p-z)$ wave field. In Figure 2a, which illustrates the result of continuation with the correct $v(z)$ function, it is clear that the relationship between the image in the wave field and the corresponding slowness trajectory (the solid line) is not trivial. Fortunately, this relationship can be predicted. As we show below, the continuation itself has certain self-stabilizing feedback properties which enable a velocity profile to be estimated accurately from the use of any arbitrary criterion for locating the slowness trajectory, provided that it is consistently applied.

In Figure $2 \mathrm{a}$ it can be seen that the optimal slowness trajectory is near, but not coincident with, the maximum amplitude locus in the imaged wave ficld. Part of the reason for this is that the $p-z$ wave field images reflection coefficients, and each of these contains a phase shift which depends upon the angle of incidence and the velocity gradient at the ray bottoming point. Specifically, if one assigns zero shift to refraction branches and precritical reflections, then wide-angle reflections have phase shifts which progress from $\pi / 2$ at the critical reflection to $\pi$ at large offsets, and any ray which touches an internal caustic receives an additional $\pi / 2$ shift. The latter occurs, for example, to rays which are refracted in a region of sufficiently high velocity gradient that a triplication is produced in the traveltime curve. The existence of these phase shifts suggests that better convergence could be obtained by identifying those $p$ ranges containing particular types of arrivals and applying appropriate phase shifts. This identification could be made in the $p-\tau$ plane since the radii of curvature of refraction and reflection $p-\tau$ loci have opposite signs (cf., Figure $1 \mathrm{~b}$ ). Alternatively, an algorithm which is independent of phase shift could be sought.
We refrain from making progressive phase shifts as a function of $p$ in the $p-\tau$ wave field, as described above. because this requires one to make a specific interpretation which may bias the inversion results. Instead, we have shifted the phase of the entire wave field by $5 \pi / 4$. The result, for the wave ficld in Figure $2 \mathrm{a}$. is shown in Figure $2 b$. This phase shift consists of three contributions. First, there is a $\pi / 2$ shift associated with the requirement that the far-field radiation condition be satisfied (cf., Aki and Richards, 1980, p. 417). Then, there is a $\pi / 4$ shift due to the fact that we are dealing with a $2-\mathrm{D}$ representation of propagation in three dimensions (Chapman. 1978). These two shifts are exact for all $p$ values. The final shift of $\pi / 2$ is the average associated with the range of reflection coeflicients expected for the various arrivals (refractions and reflections) in a typical refraction profile.

With the net phase shift of $5 \pi / 4$, the locus containing the maximum positive amplitudes (or the first significant pulse when considering real data) in an imaged wave field should cverywhere be within $\pi / 2$ of the correct slowness locus. Using this modified form of the imaged wave fields, it was straightforward to obtain convergence to the neighborhood of the correct model by using a very simple criterion for determining the approximate location of the slowness trajectory at each itcration. The exact phase shift applied is not critical since it does not enter into the end product directly. It simply alters the criterion for locating the optimal slowness trajectory relative to the $p-z$ wave field without altering the position of the trajectory itself.

Downward continuation is a stable operation, even with highly erratic velocity-depth input functions. It does not matter if slowness trajectories are located incorrectly in intermediate iterations; these effects are not cumulative from iteration to iteration because the downward continuation at each sten always starts with the original slant stacked wave field. Also, it does not matter if the criterion used to find the slowness trajectory changes from one intermediate iteration to the next. In fact, we found that when picking trajectories by hand, the picking criterion evolved from iteration to iteration, and different criteria were used over different $p$ ranges in the attempt to attain convergence. All of this is expected and is a direct consequence of the various phase shifts associated with different types of arrivals as described above. None of the evolutionary process enters or biases the final solution, provided identical picking criteria are used on the two successive output wave fields used to identify the condition of convergence. The appropriate criterion is a function of $p$ and is definable as that which produces convergence to a single slowness-depth line. This definition can be used directly to obtain convergence without any knowledge of arrival types or phase shifts.

The slant-stacked wave field in Figure Ib was chosen to illustrate the convergence of the inversion. This example was done in an interactive format, in which the slowness trajectory at each iteration was extracted by hand using the criterion of the maximum positive amplitude at each $p$. Use of this inflexible criterion precludes convergence to a single line. The results are shown in Figure 3. Beyond iterations 4 and 5 (Figures $3 \mathrm{c}$ and $3 \mathrm{~d}$ ), the solution did not converge further, but oscillated between the two positions represented by iterations 4 and 5 . This oscillation indicates a consistent bias associated with the criterion of maximum positive amplitude used for picking the slowness locus. This oscillation is a useful behavior and will be discussed below. It is encouraging that such a simple criterion produces a result close to and exhibiting the same general shape as the desired curve (Figure 3e). In fact, for many practical purposes, this level of convergence $(<2$ percent error in velocity when averaged over the profile) is already adequate. 

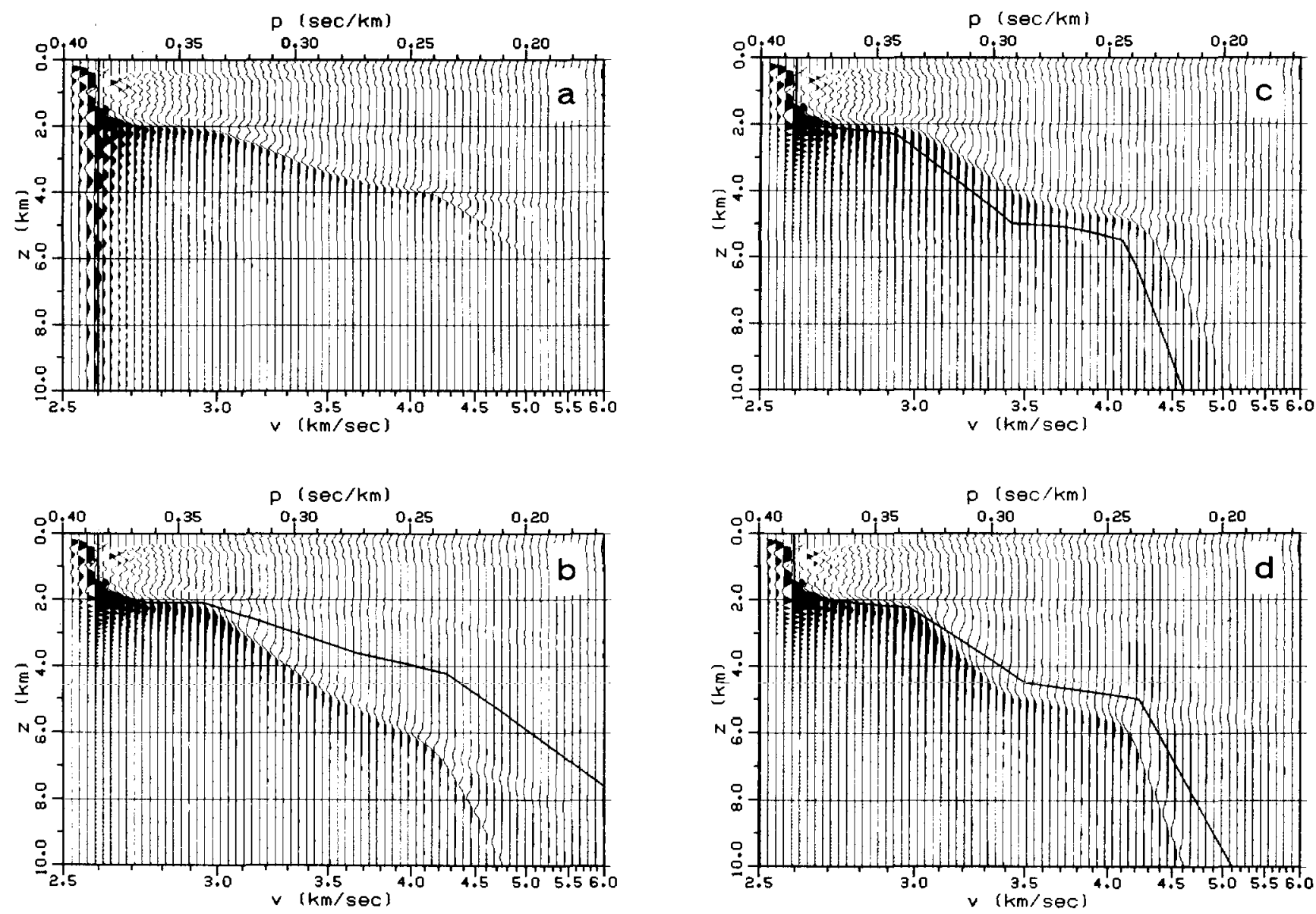

FIG. 3a, 3b. Convergence by iteration in an interactive mode. The wave field being downward continued is the plane-wave decomposition of the synthetic data shown in Figure lb. Figure 3a contains the starting $v(z)$ model, a constant velocity of $2.6 \mathrm{~km} / \mathrm{sec}$ at all depths (see the solid line), and the resultant wave field. The solid line in (b) is the slowness trajectory which was extracted by hand from the wave field in (a). Downward continuation with this slowness function produced the wave field in (b). The later stages of this inversion are shown in Figure $3 \mathrm{c}$ to $3 \mathrm{f}$.

The example in Figure 3 illustrates one approach to inversionfinding a model which is in some sense a single best-fit curve to the data. The uncertainty in such a model is indicated in the present scheme by the width of the $p-z$ image at convergence and is directly derived from the frequency content and the time resolution in the original data.

Another approach to inversion concentrates on determining the envelope of all possible models that are consistent with a given data set (cf., McMechan and Wiggins, 1972; Bessonova et al, 1974, 1976). In wave field inversion an explicit envelope can be determined by using a property of the downward continuation algorithm. If a continuation is performed with a velocity estimate that is too high at every depth, it will indicate depths that are everywhere too great. Conversely, if the continuation velocity is consistently too low, consistently shallow depths are produced. We have also seen (Figures $3 c$ and $3 d$ ) that a consistent bias produces convergence to two pseudo-stable states. These states are mutually inverse (i.e., imaging with either of the corresponding velocity functions produces the other). This idea has a num-
FIG. 3c, 3d. Similar to Figures $3 a$ and $3 b$ in that they illustrate intermediate results in the process of inversion of the slant stacked wave field in Figure $1 \mathrm{~b}$. Iteration 4 is shown in (c) and iteration 5 is shown in (d). The final results of the inversion are shown in Figures $3 \mathrm{e}$ and $3 \mathrm{f}$.

ber of implications. First, such a pair of mutually inverse functions constitutes an envelope since one is everywhere too deep and the other is everywhere too shallow. In this context, the term envelope refers to a pair of lines between which the optimal solution lies and is not to be confused with the depth resolution of a solution as indicated by the width of the $p-z$ image at convergence. Second, it implies that one need not determine sophisticated criteria for locating the slowness locus when envelopes are the primary desired result (all that is required is any picking criterion that is consistently biased). Third, a good estimate of the desired $v(z)$ function can be obtained simply by averaging those of the two states. (An example of this was presented above.) Finally, the potential now exists for complete inversion by a computer program since a simple criterion is sufficient and produces both an envelope and a reasonably accurate estimate of the velocity profile.

The interactive mode is likely the most viable form for practical application since it can avoid, through the introduction of human intuition and experience, most of the potential problems in identify- 

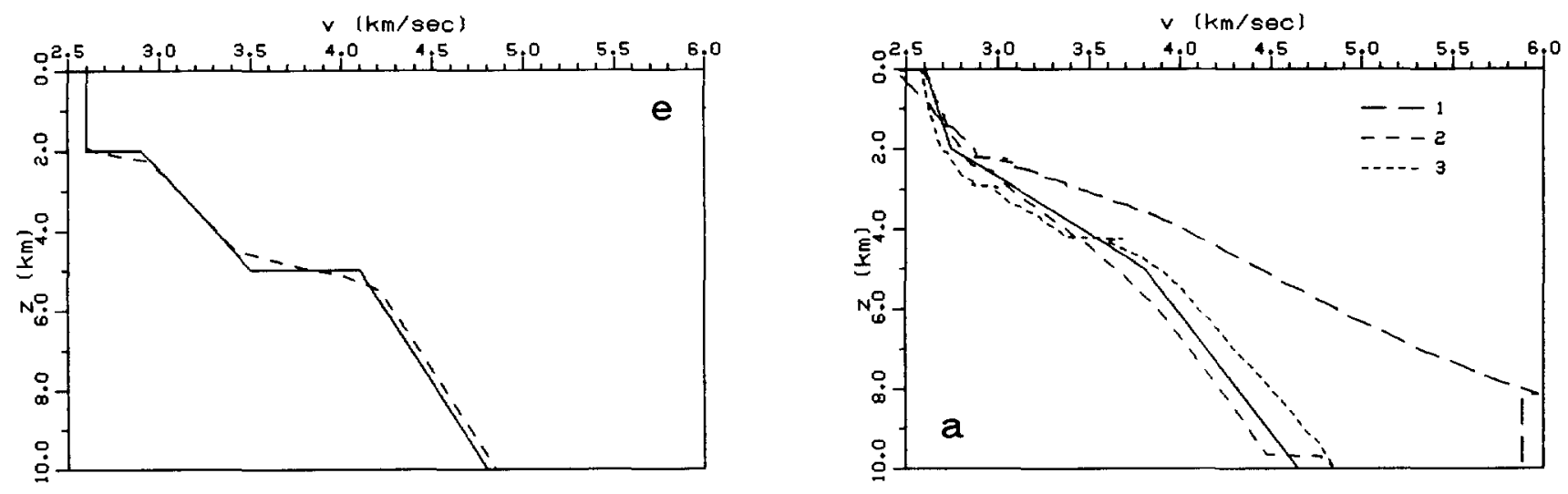

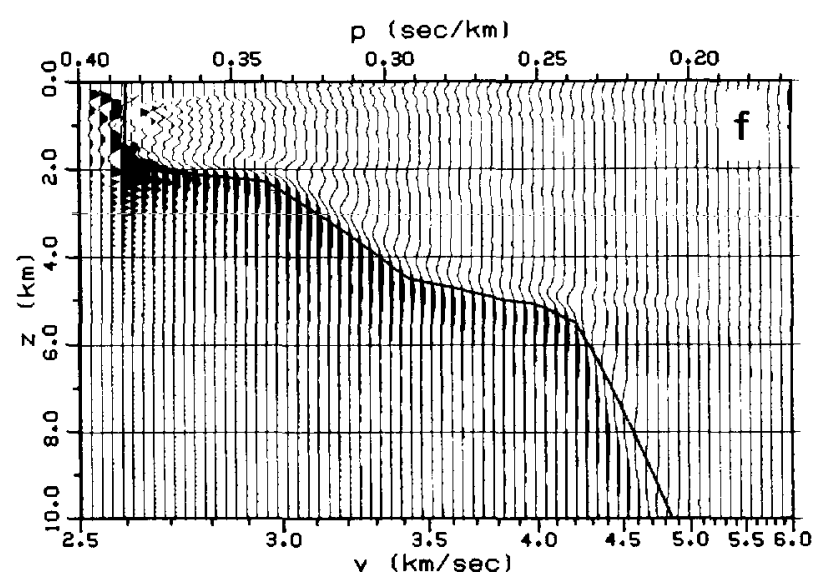

FIG. 3e, 3f. The final results of the inversion of the synthetic data wave field in Figure la. Figure $3 \mathrm{e}$ contains the average of the slowness loci of iterations 4 and 5 (the dashed line) and the correct solution (the solid line). Except for differences in the velocity gradients near 2- and 5-km depth, the obtained and desired profiles are very similar. Figure $3 f$ shows the slowness trajectory obtained by averaging the results of iterations 4 and 5 superimposed on the wave field obtained by downward continuing the slant-stacked wave field in Figure $1 \mathrm{~b}$ with this function.

ing the slowness trajectory from the artifacts. However, for certain data sets, it will be possible to do an inversion in a completely automated mode. Figure 4 contains an example of the result of an automated inversion. The solid velocity-depth line in Figure $4 \mathrm{a}$ was used to generate a synthetic refraction profile which was then slant stacked. This $p-\tau$ wave field was then iteratively continued using a constant velocity of $2.6 \mathrm{~km} / \mathrm{sec}$ as the starting model. The picking criterion for finding a slowness locus was the maximum positive amplitude at each $p$. The velocity-depth functions produced by the first three iterations are shown in Figure 4a as the dashed lines. Both iterations 2 and 3 are everywhere within $0.2 \mathrm{~km} / \mathrm{sec}$ of the correct solution. As a final estimate, the results of iterations 2 and 3 were averaged and a continuation was done with this slowness function. The output wave field of this last iteration is shown in Figure 4b. The solid line superimposed in Figure $4 \mathrm{~b}$ is the slowness function used for this iteration.

Figure $4 \mathrm{~b}$ also illustrates the effect of the failure of the algorithm used to find the slowness trajectory that would compensate for the varying phase shifts on different arrival branches. Through

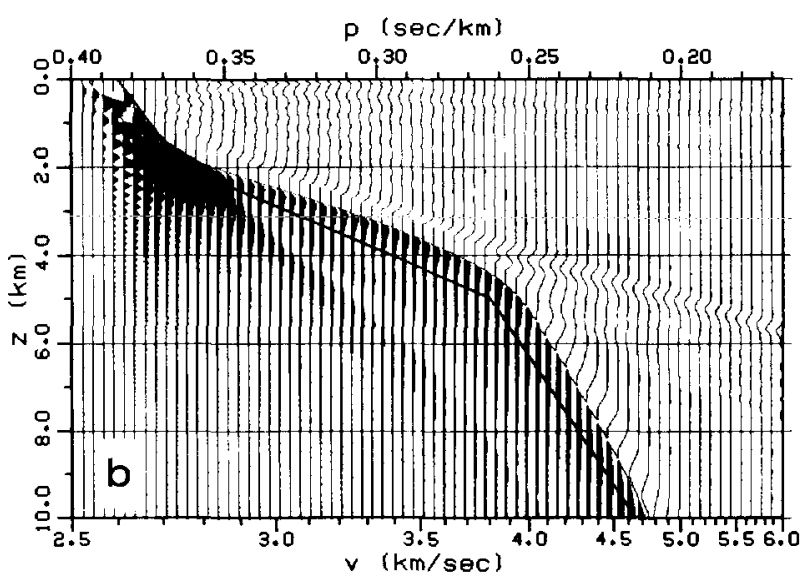

FIG. 4. Convergence in an automatic mode. The solid line in (a) is the velocity model for this example. The dashed lines are the results of the first three iterations. where at each step the slowness curve was picked automatically. The lower panel (b) shows the results of downward continuing with a velocity function (the solid line) that is the average of iterations 2 and 3 .

the downward continuation algorithm, an uncompensated phase shift at some $z$ (which in effect produces a shifted slowness estimate) leads to an offset in $z$ for all greater values of $z$. An error in picking at shallow $z$ will be present at all greater $z$, and subsequent errors are cumulative.

Although many of the examples presented here involve the use of maximum positive amplitude as a slowness picking criterion, this restriction is easy to relax. Maximum positive amplitude has been used because it is a stable criterion for producing rapid convergence to the neighborhood of the correct solution but, by itself, it cannot produce complete convergence to a single line. In general, it is sufficient to know that convergence is defined by the coincidence of the input slowness trajectory and its image. When this coincidence is obtained, no matter how devious or empirical the route, all the correct phase shifts will have been included and any envelopes obtained at previous steps will have collapsed to a single line.

Amplitude information can be used to speed convergence. For example, in Figure $2 \mathrm{a}$, the existence of precritical reflections (the 

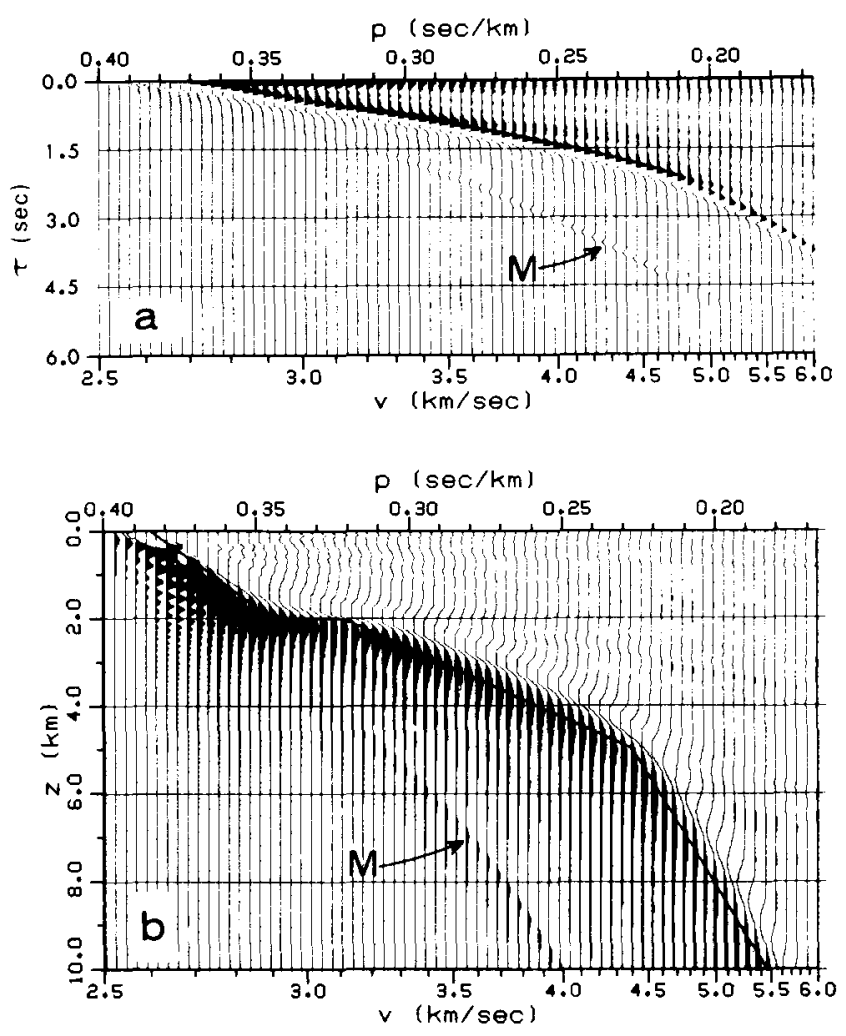

FIG. 5. The transformation of multiple reflections. First-order multiples were included in the synthetic data profile used to produce the slant-stacked wave field in (a). Migration of this wave field with the correct velocity profile [the solid line in (b)] produces the wave field in (b). In both (a) and (b) the image labeled $M$ is the first multiple.

extension of the image through lower slowness values from the critical reflections at the "knees" of the image) indicates the presence of a significant velocity contrast. The use of such additional information would produce better estimates of appropriate slowness trajectories. Additionally. where a true reflection exists. the associated progressive phase shifts are evident in the output wave field (cf., Figures $2 \mathrm{a}$ and $2 \mathrm{~b}$ ). Another subjective aid is inherent in the level of focusing of the slowness image in the imaged wave field. The image becomes better focused as the correct velocity estimate is approached (compare Figures $3 \mathrm{a}$ and $3 \mathrm{~b}$ ). The reason that amplitude constraints (e.g. . the increased amplitude associated with a critical reflection) enter the solution only in a subjective way is that the present form of wave field inversion is still based on integral constraints (in the form of traveltimes) on the velocity-depth function. Amplitude information is an expression of the behavior of velocity gradients and so provides derivative constraints. In order to make use of such information the data must be recorded so that true amplitudes may be recovered, and a prestack scaling of data by $(2 h)^{-12}$ can be included to compensate for geometrical spreading (cf.. Phinney et al. 1980).

Certain types of data, such as typical marine profiles and the Imperial Valley data presented below, contain prominent multiple reflections. Since these arrivals often mask primary arrivals, their removal is the topic of much research. The transformations discussed here may be applied to this problem. Figure 5 a contains the plane-wave decomposition of a synthetic data profile consisting of both primary arrival branches and their first free-surface multiple branches. In the $p-\tau$ plane. the energy in the multiple is separated from the primary energy since, for each $p$, it plots at twice the $\tau$ of the primary. Similarly, in the imaged wave field in Figure 5b. the primary and multiple images are separated.

Note this approach to multiple removal is also valid for data collected over structures other than flat layers. The transformations themselves do not contain any restricting assumptions about the origin of the data (slant stacking does not involve any velocity assumptions, and even migration at a grossly incorrect velocity would still separate primaries and multiples). There are, however. still problems where a precritical reflection branch of a multiple crosses a primary branch. An alternate approach to multiples attempts to utilize the information present in them. We leave migration of multiples for future consideration

The presence of a low-velocity zone in the velocity model will introduce an unknown which cannot be determined by examining refracted arrivals alone. The traveltime shadow zone associated with a low-velocity zone (in the $t-h$ domain) becomes transformed into a jump in $\tau$ at constant $p$ in the $p-\tau$ domain. The subcritical reflections may provide the necessary information to determine the structure of the low-velocity zone.

Finally, it is of theoretical interest that we have empirically shown there is a direct correspondence between Wiechert-Herglotz inversion and downward continuation. We expect that an asymptotic or stationary phase form of the inversion equation (to delete the $\omega$-dependence) can formally be shown to be equivalent to the Wiechert-Herglotz integral. Also, these concepts are expected to be applicable to the inversion of surface waves as well as body waves since it is possible. with certain assumptions, to invert a dispersion curve with the Wiechert-Herglotz integral (Takahashi. 1955: Nolet and Kennett. 1978). These topics are the subjects of current investigation.

\section{APPLICATION TO RECORDED DATA}

In order to evaluate the applicability of wave field inversion to actual field data, a digitized refraction profile of high spatial density was obtained from the United States Geological Survey (USGS). These data, which are presented in Figure 6a, were recorded in the Imperial Valley of southern California in 1979.

With one important exception, the analysis of the Imperial Valley data followed closely the interactive procedure outlined above in the analysis of the synthetic data of Figure la. The exception is that the slowness picking criterion was not fixed. but was allowed to change progressively so the solution converged to a single line rather than an envelope. The data were slant stacked to produce the $p-\tau$ wave field shown in Figure 6b. At each iteration, the slowness trajectory was extracted by hand from the imaged wave field. Convergence was obtained in four iterations. In Figure $6 \mathrm{c}$. the velocity-depth profiles corresponding to the second and the final (the fourth) iterations. are shown. Also in Figure $6 c$ is the velocity profile obtained independently by $W$. Mooney of the USGS from a detailed ray-tracing analysis of an earlier reversed refraction line in the same area. This previous line was approximately parallel to the one analyzed here; the shotpoint for the later profile also corresponded nearly exactly to the previous shotpoint. Thus, it is reasonable to make a general comparison of the results of the two studies as presented in Figure $6 c$.

Figure 6d contains the slowness trajectory extracted from the imaged wave field of the fourth iteration. This line is superimposed upon the wave field obtained by downward continuing with it to illustrate the convergence condition. The slowness locus is particularly clear in this wave field. 

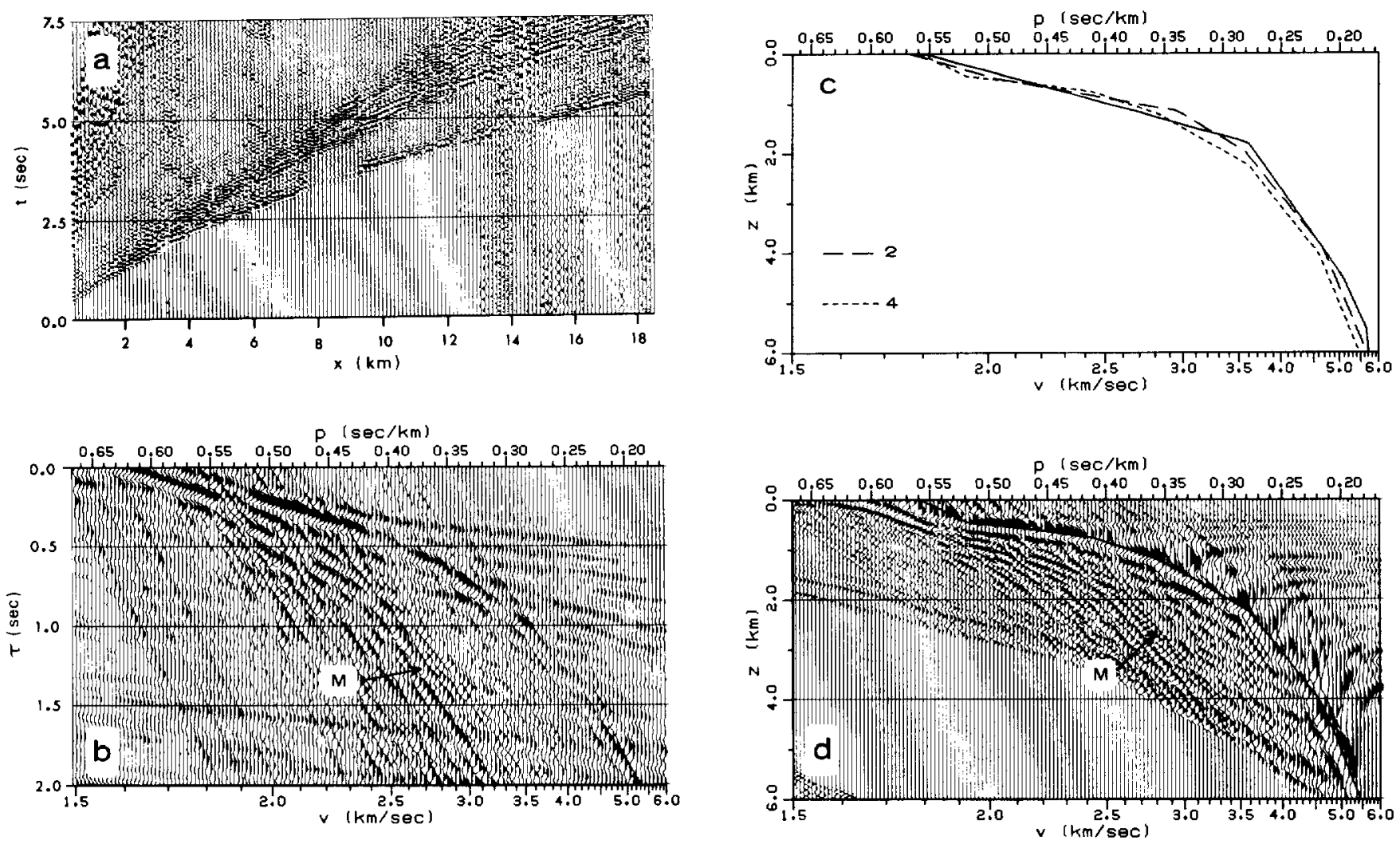

FIG. 6a. 6b. Processing of real data. The record section in (a) was recorded in the Imperial Valley by the USGS. For this figure, each seismogram was scaled to have the same maximum amplitude and is plotted at approximately its correct offset (the actual spacing was not constant and there were a few bad traces that were not included). For the slant stack, the actual offset of each trace was used. The slant-stack wave field is shown in (b). The result of inversion of these data by downward continuation is shown in Figures $6 \mathrm{c}$ and $6 \mathrm{~d}$. The image labeled $\mathrm{M}$ in (b) corresponds to the first multiple (PP).

There are some features of this Imperial Valley example which illustrate points made in the theoretical discussion above. For example, the data (Figure 6a) contain two regions of decreased resolution in the first arrival branch, one between 7 - and 9-km offset where there is a decrease in amplitude, and one between 13 and $15-\mathrm{km}$ offset where there is an apparent increase in the noise level. These two regions can be seen in transformed form in both the slant-stacked and downward-continued wave fields where they indicate an increased local uncertainty in $\tau$ and $z$, respectively. From the width of the slowness image, the depth resolution is estimated to be of the order of $0.1 \mathrm{~km}$ on the average, with better resolution at those depths associated with clear arrivals in the data and worse at those depths associated with noisy and less coherent arrivals. The prominent free-surface multiple PP, which can be seen in the data, is also transformed into the $p-\tau$ and $p-z$ domains-as predicted in Figure 5.

During the inversion of the recorded data, the focusing observed with the synthetic data was very noticeable. As the correct velocity profile was approached, the sharpness of focus of the slowness trajectory approached that seen in the slant stacked wave field.

In comparing Figures $6 \mathrm{~b}$ and $6 \mathrm{~d}$, there are some $p-\tau$ loci
FIG. 6c, 6d. The result of inversion by wave field transformation of the data in Figure 6a. The two dashed lines in (c) are the slowness trajectories for iterations 2 and 4 . The solid line is the result of inversion (by detailed ray tracing) of a reversed refraction profile near the line along which the data in Figure $6 a$ were recorded. The lower (d) part illustrates the relationship between the slowness locus (the solid line) and the output wave field at convergence. The image labeled $M$ in (d) corresponds to the first multiple (PP).

which seem to image to horizontal lines. These are located near $1.5-\mathrm{km}$ depth and may be precritical reflections from small velocity contrasts, but we see no evidence for any large velocity discontinuities in this profile.

In this section the inversion of refraction data by wave field transformations has been demonstrated by the processing of a real data set from the Imperial Valley, California. These preliminary results encourage further application and development of the method.

\section{CONCLUSIONS}

We have presented an alternate method for inversion of spatially dense refraction data that is based on the technique of wave field continuation. The main advantages of the method are that the entire wave field is present throughout the inversion and the desired feature (the velocity-depth curve) is extracted directly from its image in the output wave field. This eliminates the subjective bias which can occur when traveltime or tau curves are picked. The method is robust and is self-consistent in the sense that a consistent bias in extracting the image will produce convergence to a bistable state which envelops the optimal solution. The depth resolution is indicated by the width of the $p-z$ image and is 
derived directly from the frequency content and the time resolution in the original data. The method has been used, apparently successfully, to invert a data set recorded in the Imperial Valley, California.

\section{ACKNOWLEDGMENTS}

During the course of this project, one of the authors (G.M.) was on leave at the Stanford Exploration Project, Dept. of Geophysics, Stanford University, which provided superb computing facilities. Critical reviews of the paper by R. Stolt, C. Chapman, M. Yedlin, W. Mooney, and D. Hill were much appreciated. The Imperial Valley refraction data were kindly provided by the USGS, one of the sponsors of the Stanford Exploration Project. In particular, the aid of J. Healy, W. Mooney, W. Kohler, and J. Roloff of the USGS was invaluable. The work was supported in part by the Department of Energy, Mines and Resources, Canada. Contribution from the Earth Physics Branch no. 868.

\section{REFERENCES}

Aki, K., and Richards, P. G., 1980, Quantitative seismology, theory and methods, vol. 1: San Francisco, W. H. Freeman and Company.

Bessonova, E. N., Fishman, V. M., Ryaboyi, V. Z., and Sitnikova, G. A., 1974. The tau method for inversion of traveltimes-I. Deep seismic sounding data: Geophys. J., v. 36, p. 377-398.

Bessonova, E. N., Fishman, V. M., Shnirman, M. G., Sitnikova, G. A. and Johnson, L. R., 1976, The tau method for inversion of traveltimes-II. Earthquake data: Geophys. J., v. 46, p. 87-108.
Bracewell, R. N., 1956, Strip integration in radio astronomy: Aust. J. Phys., v. 9, p. 198-217.

Bracewell, R. N., and Riddle, A. C., 1967, Inversion of fan-beam scans in radio astronomy: Astrophys. J., v. 150, p. 427-434.

Chapman, C. H., 1978, A new method for computing synthetic seismograms: Geophys. J., v. 54, p. $481-518$.

Claerbout. J. F., 1976, Fundamentals of geophysical data processing: New York, McGraw-Hill Book Co., Inc.

Garmany, J., Orcutt, J. A., and Parker, R. L., 1979, Traveltime inversion -A geometrical approach: J. Geophys. Res.. v. 84, p. 3615-3622.

Gazdag. J., 1978, Wave equation migration with the phase shift method: Geophysics, v. 43, p. 1342-1351.

Gel'fand, I. M. Graev, M. I., and Vilenkin, N. Ya., 1966, Generalized functions, v. 5, Integral geometry and representation theory: New York, Academic Press.

McMechan, G. A., and Ottolini, R. 1980. Direct observation of a $p-\tau$ curve in a slant stacked wavefield: SSA Bull., v. 70, p. 775-789.

McMechan, G. A., and Wiggins, R. A. 1972, Depth limits in body wave inversions: Geophys. J., v. 28, p. 459-473.

Nolet, G., and Kennett, B., 1978, Normal-mode representations of multiple-ray reflections in a spherical Earth: Geophys. J., v. 53, p. 219-226.

Phinney, R. A., Chowdhury, K. R.. and Frazer, L. N., 1980, Transformation and analysis of record sections: preprint.

Schultz, P. S., 1976, Velocity estimation by wavefront synthesis: Ph. D. thesis, Stanford University.

Schultz, P. S., and Claerbout, J. F., 1978, Velocity estimation and downward continuation by wavefront synthesis: Geophysics, v. 43, p. $691-$ 714.

Stoffa, P. L., and Buhl, P. 1979, Two-ship multichannel scismic experiments for deep crustal studies: J. Geophys. Res., v. 84, p. 7645-7660.

Takahashi, T., 1955, Analysis of dispersion curves of Love waves: Bull. Earthq. Res. Inst., v. 33, p. 287-296. 